\title{
Effect of the tensile strength on the stability against rotational breakup of icy bodies
}

\author{
Imre Toth ${ }^{1}$ and Carey M. Lisse ${ }^{2}$ \\ ${ }^{1}$ Konkoly Observatory \\ Postbus 67, H-1525 Budapest, Hungary \\ email: tothi@konkoly.hu \\ ${ }^{2}$ Johns Hopkins University Applied Physics Laboratory \\ 11100 Johns Hopkins Road, Laurel, MD 20723-6099, USA \\ email: carey.lisse@jhuapl.edu
}

\begin{abstract}
Focusing on primitive icy minor bodies in the solar system like cometary nuclei, centaurs, transneptunian objects (TNOs), and main-belt comets (MBCs) we investigate the stability of these objects against rotational breakup by comparing their location in (radius rotational period) space with respect to separation lines of the stable and breakup zones in this plane. We estimate the bulk tensile strength according to new structural and elasto-mechanical models of grain-aggregates, using these tensile strengths to compute separation lines. We note that the process of grain coagulation and growth is highly uncertain in the field of solar system formation and we simply don't know how to grow interstellar grains to aggregates larger than about $1 \mathrm{~mm}$ but we apply in our calculations the recently available elasto-mechanical models of grain-aggregates. Accorging to this study most of the observed comets, centaurs, TNOs, and MBCs are stable against rotational breakup, with a few notable exceptions. E.g., we suggest that the rotational fission is a likely scenario for the Haumea-family in the Kuiper belt.
\end{abstract}

Keywords. solar system: general, solar system: formation, comets: general, comets: individual (133P/Elst-Pizarro), Centaurs, Kuiper Belt, Haumea-family, asteroids, Main-Belt Comets

\section{Introduction and overview}

The physical characteristics of primitive minor bodies in the solar system like bulk interior properties and mechanisms and ingredients of cometary emission activity, are very complex and have not been well understood yet (e.g., Weissman \& Lowry 2008; Belton 2009; Biele et al. 2009; Jewitt 2009). For example, we have little knowledge of mechanical properties like the tensile strength of the interior material of primitive small bodies like comets, related asteroids, and dwarf planets. We note that there are different strength definitions throughout the literature (Holsapple 2009). Here we are concerned with the influence of the static tensile strength of the material on the stability against rotational breakup of primitive minor bodies.

Up to now the following methods have been used to derive the tensile strength of primitive small bodies of the solar system: (i) examining the observed rotational stability, (ii) modeling the splits due to tidal forces (e.g., tidal breakup of sungrazing comets, and D/Shoemaker-Levy 9 disrupted by Jupiter), (iii) modeling results from the Deep Impact experiment(to determine the dynamic and estimate the static tensile strengths), (iv) prediction from theory. The best estimate of tensile strength of cometary nuclei ranges from $10 \mathrm{~Pa}$ to $100 \mathrm{kPa}$ (Jewitt 1992; Boehnhardt 2004; Weissman et al. 2004, and references therein).

Formerly it was common wisdom that cometary interiors consist of materials there is extremely porous structure and low strength (see review by Weissman et al. 2004). 

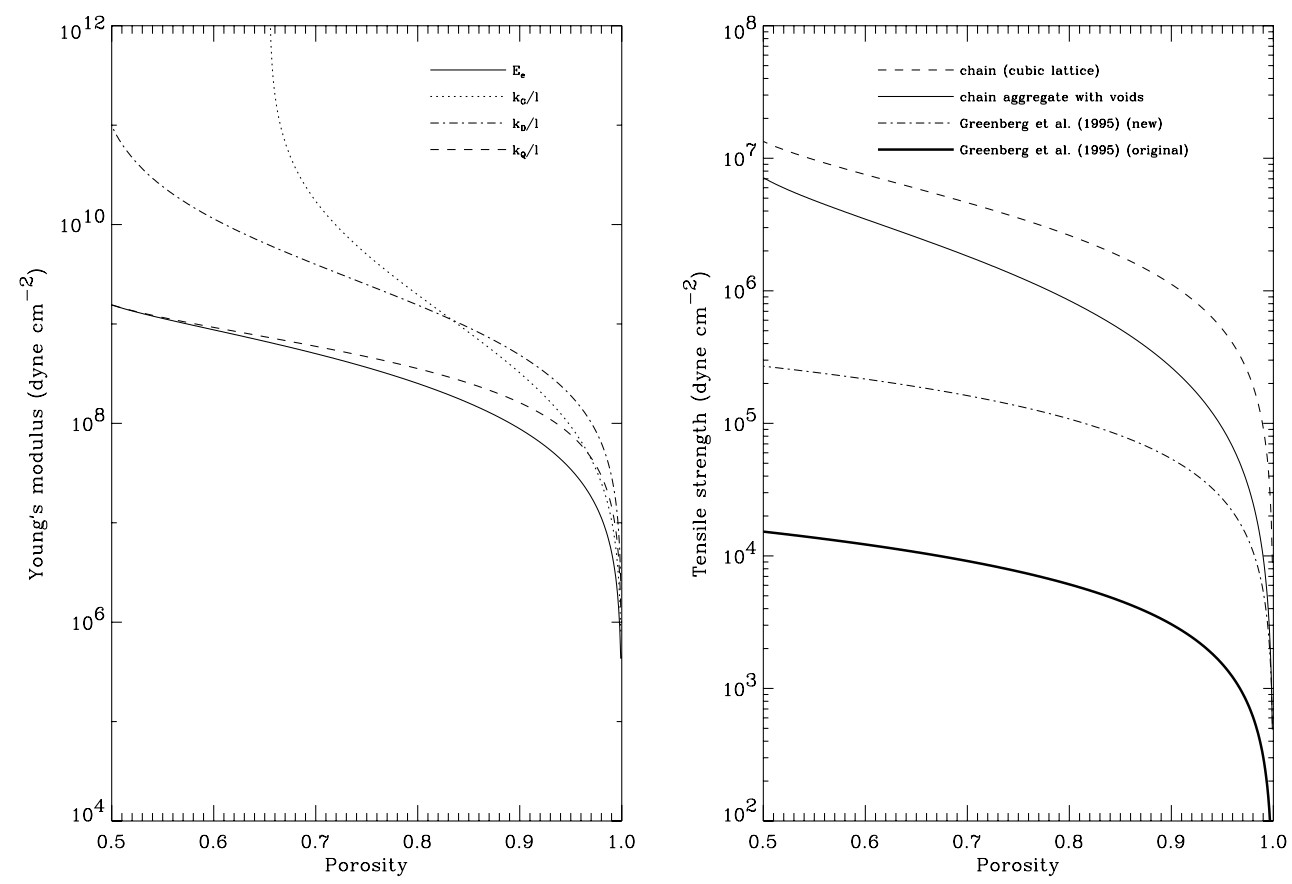

Figure 1. Effective Young's modulus (left panel) and tensile strength (right panel) of different aggregate models are displayed. Effective Young's modulus of an aggregate (solid line) and the deformation coefficients of rolling, sliding, and stretch are shown as dotted line, dash-dotted line and dashed line, respectively. Tensile strength of cubic lattice aggregate chain (dashed line), chain aggregate with voids (solid line), Greenberg et al. (1995) model with new value of the intermolecular energy (Sirono \& Greenberg 2000), (dash-dot line), and the original aggregate model described by Greenberg et al. (1995). (dash-dot-dot line) are shown. Note that both the Young's modulus and tensile strength are rapidly decreasing for large porosities $(\gtrsim 0.7)$.

However, hitherto we have less knowledge on the bulk interior physical properties of small primitive bodies, like elastic parameters. Despite the in-situ nucleus Deep Impact space experiment, we still have limited knowledge of the bulk interior physical properties. While Richardson et al. (2007) placed limits of $10^{4} \mathrm{~Pa}$ on the effective bulk tensile strength of the comet $9 \mathrm{P} /$ Tempel-1 nucleus assuming a gravity dominated cratering mechanism, Holsapple \& Housen (2007) concluded that "the observations are unlikely to answer the questions about the mechanical nature of the 9P/Tempel 1 surface". Even after the insitu flybys the comets remain the poorest understood solar system objects. And according to Biele et al. (Biele et al. 2009), it appears doubtful that the tensile strength is as low as originally published for the nucleus of comet $9 \mathrm{P} /$ Tempel 1.

One approach to estimate the tensile strength of a rotating body is the examination of its rotational breakup stability or instability. For this purpose the early studies considered the stability of a rotating sphere or a homogeneous prolate ellipsoid rotating about its shortest axis, balancing the centrifugal and gravitational forces at the tips of the longest axis (Jewitt \& Meech 1988; Luu \& Jewitt 1992; Weissman et al. 2004).

This simplified model is a first guess to indicate the rotational instability because it describes material shedding from the elongated body surface at the tips, not a complete disintegration of the body as a whole. More complex numerical models like N-body simulations and hydrocodes now exist to follow rotational breakup for both rubble piles and 
continuum structures with and without internal cohesive forces (Weissman et al. 2003; Walsh et al. 2008; Richardson et al. 2008, 2009). But in order to easily compare models with observations there are simpler descriptions of the rotational breakup stability. One of these is based on the Drucker-Prager model and was successfully applied by Holsapple (2007) for both small rotators and large transneptunian dwarf planets like 136198 Haumea (2003 EL61) and 20000 Varuna (2000 WR106).

Another recent approach was developed by Davidsson $(1999,2001)$ for rotating spheres and biaxial ellipsoids. Applying the Davidsson models Toth \& Lisse (2006) explored the regions of stability, fragmentation, and destruction for cometary bodies versus rotational breakup in the radius - rotational period plane. In this work we are continue this line of investigation, and present new results taking into account the elasto-mechanical parameters of new grain-aggregate models. We then compare the location of well observed objects like comets, centaurs, transneptunian objects, and a new class of primitive minor bodies, the main belt comets to the predicted regions of stability and disintegration.

\section{Outline of the method and analysis}
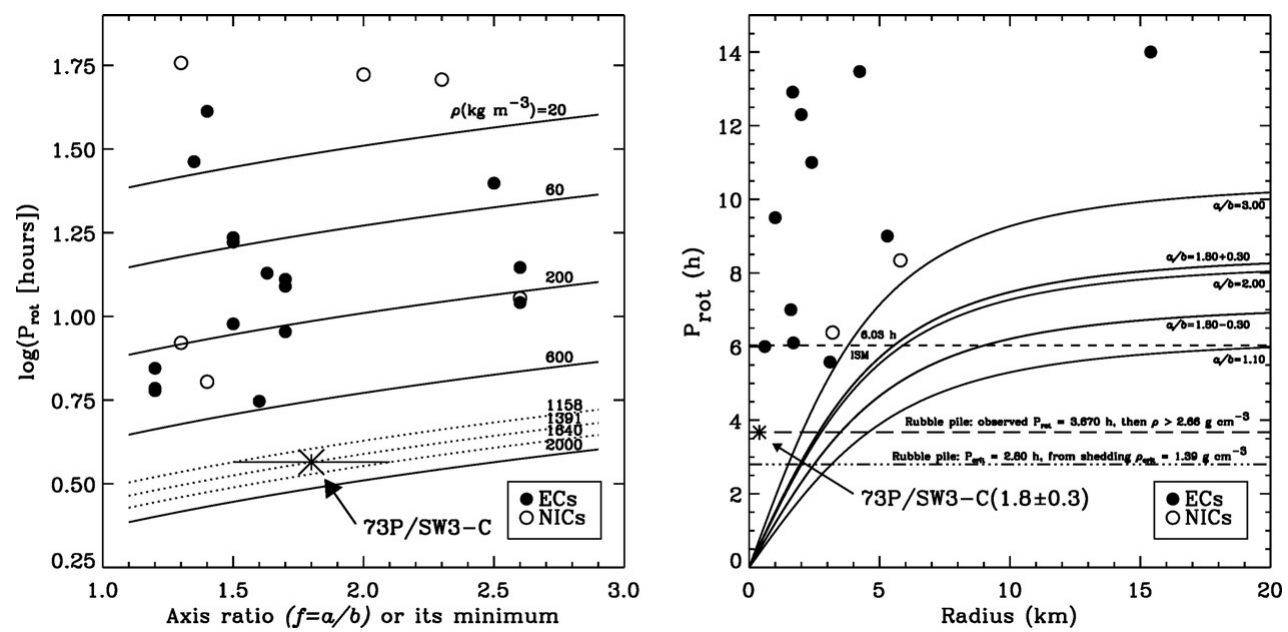

Figure 2. The fragment $\mathrm{C}$ of comet $73 \mathrm{P} / \mathrm{Schwassmann}-$ Wachmann 3 is a fast rotator and material shedding from its surface possible (left panel) but the body itself is safe against the rotational breakup (right panel). Set of curves correspond to bulk density of the boady (left panel) and the curves of axial ratios of a rotating prolate body are shown (right panel). Ecliptic comets (filled dots) and nearly-isotropic comets (open circles) are also displayed.

Bulk material parameters are a consequence of planetesimal or cometesimal formation history. According to the formation scenario for planetesimals and cometesimals, the sub-kilometer to kilome-ter-sized planetary and minor body precursors form due to nonelastic collisions between dust agglomerates in combination with adhesive surface forces (Greenberg et al. 1995; Sirono \& Greenberg 2000; Weidenschilling 2004; Weissman et al. 2004, and references therein). The process of grain coagulation determines the resulted aggregate structure, bulk density, porosity, effective Young's modulus, and tensile strength of grain-aggregates. But note that the process of grain coagulation and growth is highly uncertain in the field of solar system formation and we simply don't know how to grow ISM grains to aggregates larger than about $1 \mathrm{~mm}$. Collisions in the midplane of larger aggregates should be destructive by all measures and see the recent work of Chiang \& Yudin (2009). 
Here we apply the newer models of grain-aggregate model mechanical properties for the interior material properties of bulk nuclear material. This problem is certainly not solved by Sirono \& Greenberg (2000) but we apply it because i) it is a recently available elasto-mechanical model of primitive small body-forming grain-aggregates, and ii) this model predicts a stronger material which is suspected by some recent studies (Holsapple 2007; Biela et al. 2009).

Aggregate material tensile strength. We calculate the effective Young's modulus and derive the tensile strength grain-aggregate models following Sirono \& Greenberg (2000). With the use of elastic constants for rolling, sliding, and stretching displacements of a grain, based on to the formulas obtained by Dominik \& Tielens (1997), the effective Young's modulus of the aggregate is computed.

We note that most recently the reality of the grain-aggregate models are also tested in laboratory and confirmed by new calculations and comparison with some observations (Blum et al. 2006). In our approach we calculate the tensile strength for pure chain aggregates and cubic lattice structured aggregates. The derived effective Young's modulus and tensile strength of different aggregate models are displayed in Fig. 1. Note that both the Young's modulus and tensile strength are rapidly decreasing for large porosities (porosity $\gtrsim 0.7$ ) for all aggregate models considered here.

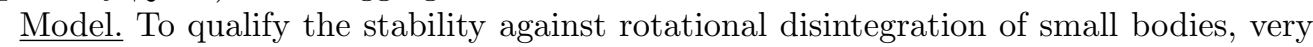
useful, user friendly, and realistic models were developed by Davidsson $(1999,2001)$ for both spheres and biaxial ellipsoids, which are realistic enough to apply for minor bodies and compare them with observed sizes and rotational periods. Despite that the models are based on simplified assumptions (internal homogeneity and incompressibility) they are very good approaches to the problem. Using his formalism, the model problem reduces to the question of the appropriate choice of the material strength and bulk density parameters, taking into account the typical material parameters for primitive minor bodies. Toth and Lisse (2006) addressed the question of the internal material dependence of the rotational breakup, namely how the critical spin periods are influenced by the tensile strength for various grain-aggregate structured models. By testing different plausible physical models of the bulk material parameters of the primitive bodies the plane is divided into three segments: allowed, damaged, and forbidden regions and we investigate the location of selected primitive small bodies with respect to these zones separated by lines corresponding to the elastic parameters of grain-aggregates.

Observational data Recent surveys of the physical properties of cometary nuclei, centaurs, transneptunian objects, and main-belt comets are used to compare the expected regions of stability to the observed populations of these primitive minor bodies. In the size determinations the typical relative uncertainty is roughly order of $\sim 10 \%$, and the accuracy of rotational periods is on the order of $0.25 \mathrm{hr}$.

\section{Results and discussion}

Comets, centaurs, and transneptunian objects. Split comets are very important to understand the deep interior structure of the cometary nuclei via observing and physical characterization of the fragments (Boehnhardt 2004). We examined the rotational breakup stability of the fragment $\mathrm{C}$ of split comet $73 \mathrm{P} /$ Schwassmann-Wachmann 3 (73P/SW3-C) investigating its location in the radius - rotational period diagram. The size and period were determined from the Hubble Space Telescope observations made in April of 2006 when the comet was in favorable visibility conditions close to Earth (Toth et al. 2006, 2008). We applied two approaches: the balance between gravitational and centrifugal forces at the tip of longest body axis of a rotating prolate ellipsoid and the model developed by Davidsson (2001). Figure 2 displays the results obtained by these 

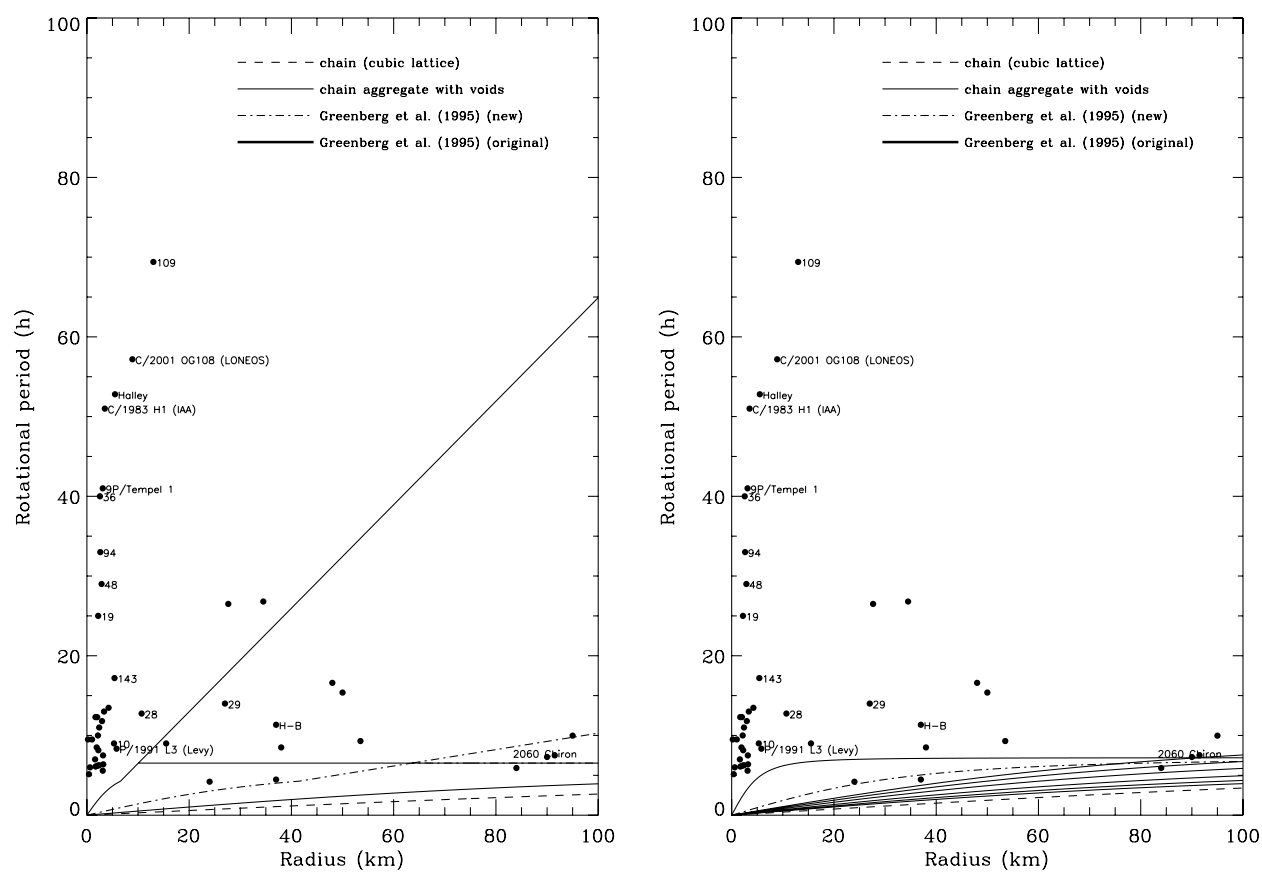

Figure 3. Comets and centaur Chiron in the radius - rotational period plane are shown (dots). Left panel: separation lines of allowed, damaged, and forbidden zones are computed for spheres according to the model given by Davidsson (1999). Right panel: set of separation lines corresponding to axial ratios (ranging from 1.01 to 3.0) of allowed/forbidden zones are computed for prolate ellipsoids according to the model given by Davidsson (2001) but only for the grain-aggregate model with voids (Sirono \& Greenberg 2000).

methods. We conclude that material shedding from the surface of this fragment is possible (left panel of Fig. 2) but despite its rapid rotation the fragment is stable against the complete rotational fission (right panel of Fig. 2).

Regarding a larger sample of comets and centaurs, for which both size and rotational period data are known the range of constituent material parameters of various grain-aggregate models we found that (i) the observed comets are in the allowed region, (ii) comet $\mathrm{C} / 1995 \mathrm{O} 1$ (Hale-Bopp) resides in the damaged region where the body is fractured and only held together gravitationally, (iii) Comet C/1996 B2 (Hyakutake) observed to emit fragments close to its perihelion and perigee in 1996, may be near the boundary of the damaged region, (iv) split comet C/1999 S4 (LINEAR) was solidly in the rotationally allowed region, making its disintegration in July 2000 due to centrifugal forces unlikely.

In contrast to the comets, the centaurs do not cluster in the allowed region, with the majority falling instead into the rotationally damaged region region for the weakest grain-aggregate models (Greenberg et al. 1995), but for stronger materials they are stable against the rotational breakup. These bodies thus seem to have different bulk physical properties than cometary nuclei.

Transneptunian objects are displayed in Figure 4. The large majority of them prove stable against rotational breakup, assuming any material property model. Only (150642) 2001 CZ31 and 135108 Haumea (2003 EL61) are definitely in the forbidden zone according to the model criteria (Davidsson 2001). Haumea is particularly intriguing, because it is a very large TNO with two small satellite companions and is the largest member of the first 


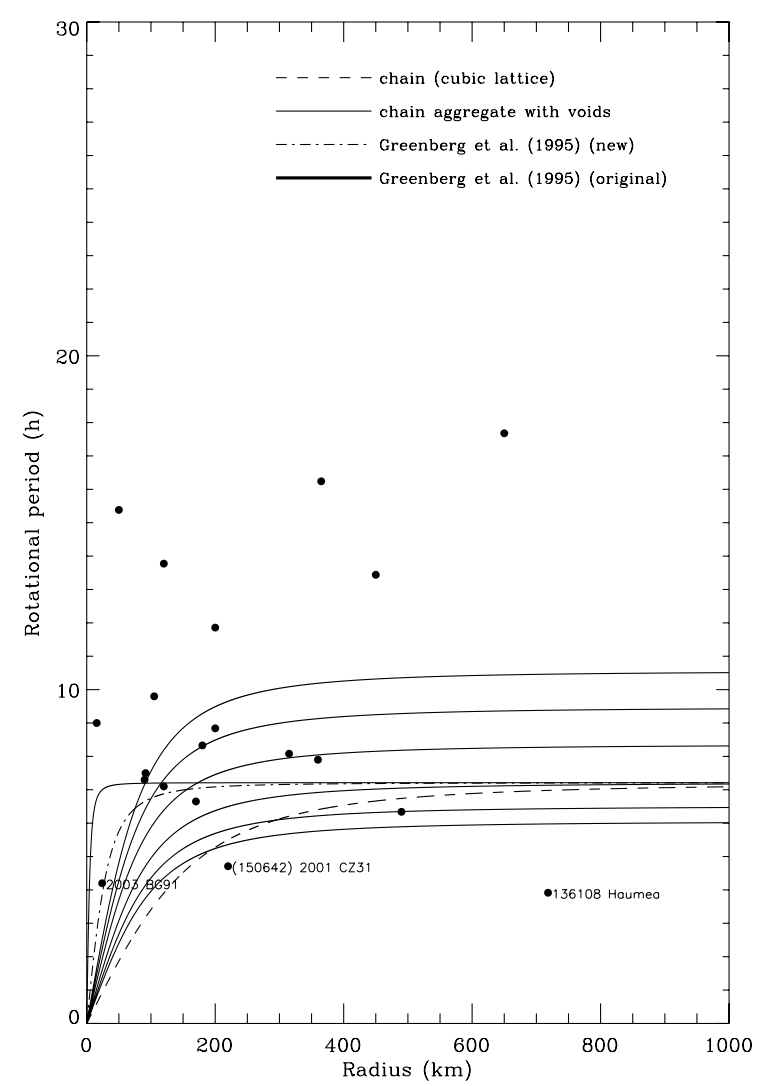

Figure 4. Transneptunian objects in the radius - rotational period plane are shown (dots). Set of separation curves (solid lines) corresponding to axial ratios (ranging from 1.01 to 3.0) of allowed/forbidden zones are computed for prolate ellipsoids according to the model given by Davidsson (2001) but only for the grain-aggregate model with voids (Sirono \& Greenberg 2000). Separation lines for other grain-aggregate models are computed for the nearly-spherical axial ratio of 1.01 .

collisional family in the Kuiper belt. The new solid body model developed by Holsapple (2007) is able to stabilize Haumea against rotational breakup assuming size- and depthdependent tensile strength of a stronger icy material. On the other hand according to the most recent analysis by Schlichting \& Sari (2009) the scenario of collisional origin of Haumea's family is doubtful. They argue that the velocity dispersion of fragments is too small to support a collision formation model. According to the close proximity to the rotational breakup limit of this large TNO in the frames of models by Davidsson (2001) and Holsapple (2007), we suggest that the mechanism of rotational fission is a likely formation scenario for this dynamical family.

Main-Belt Comets The existence of a population of comets residing in the outer region of the main asteroid belt was discovered by Hsieh \& Jewitt (2006). The MBCs occupy dynamically asteroidal orbits decoupled from Jupiter in the main asteroid belt but show cometary activity with gas-driven mass loss. The existence of the MBCs lends new support to the idea that main belt objects could be a major source of terrestrial water. If so, the physical characterization of the MBCs is extremely important. Four MBCs are currently known: 133P/Elst-Pizarro (7968 = 1996 N2), 176P/LINEAR (also $118401=$ 1999 RE70), P/2005 U1 (Read), and P/2008 R1 (Garradd). In order to examine their 


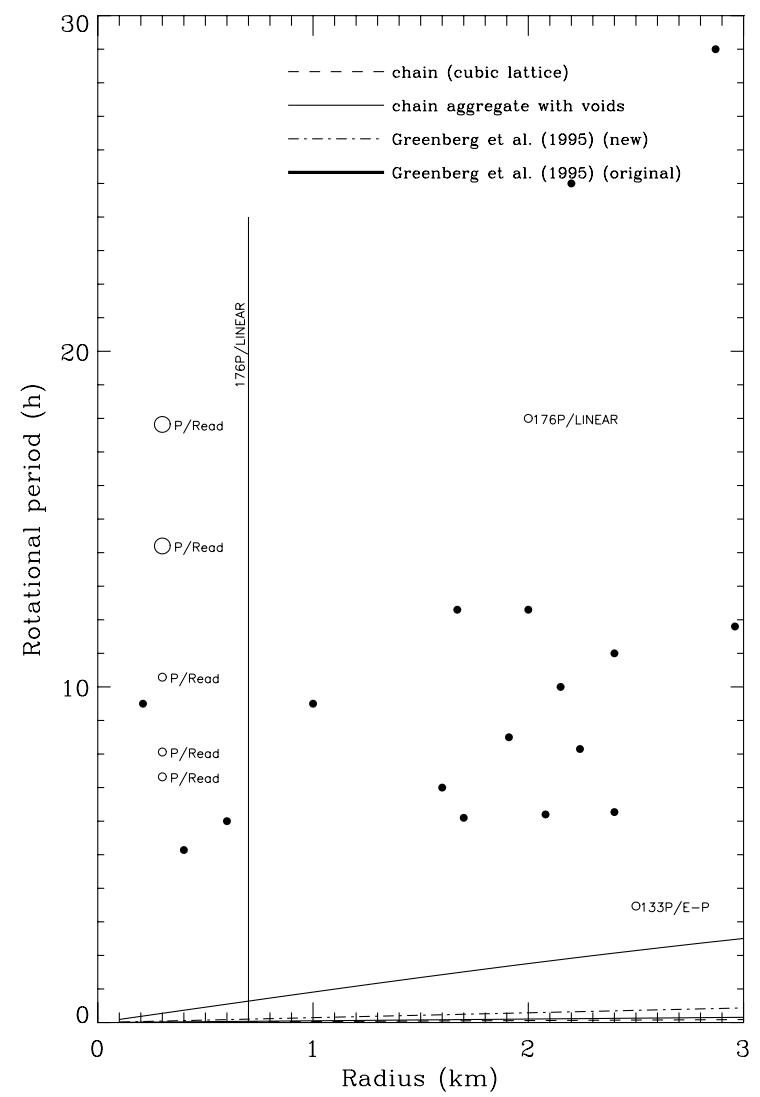

Figure 5. Main-Belt Comets with known size and rotational period in the radius - rotational period plane (open circles). The rotational period of P/2005 U1 (Read) has not been determined yet unambiguously therefore all of the proposed alternatives are shown along the vertical line. Separation lines of allowed/forbidden zones are computed for spheres according to the model given by Davidsson (1999). 133P/Elst-Pizarro is close to the rotational breakup limit for the weak grain-aggregate material (Greenberg et al. 1995). Ecliptic comets falling in the diagram are also indicated for a comparison (filled dots).

stability against rotational disintegration we adopted the observed rotational period and size data from Hsieh et al. (2004) for 133P, Hsieh \& Jewitt (2006), and Hsieh et al. (2009a) for 176P, and Hsieh et al. (2009b) for P/2005 U1. For P/2005 U1 (Read) the rotational period has not been determined yet unambiguously and the proposed periods are 14.20, 17.82, 7.32, 8.06, 10.20 hr (Hsieh et al. 2009b), all of which are shown in Fig. 5. The size and rotational period of P/2008 R1 (Garradd) are still unknown.

Justification of the validity of the application of grain-aggregate models for MBCs is based on (i) their cometary nature, (ii) the MBCs orbit in the Themis-zone in the cold icy reservoir of the outer main belt and owing to the presumably collisional origin of MBCs from a larger icy parent body, the dusty-icy aggregate structure can be assumed, (iii) the example of the D/Shoemaker-Levy 9 as a probable escaped Hilda-zone asteroid (or a temporarily resident comet).

In the radius - rotational period diagram we observe that all of the MBCs are stable against the rotational disintegration including 133P (Fig. 5). Elst-Pizarro is a fast rotating small body and it is very close to the rotational breakup limit but this object is also 
stable against rotational splitting even for the weakest material of the grain-aggregate model (Greenberg et al 1995). The two other MBCs are far from the forbidden zones for all of the aggregate material models.

The idea that the activity of the MBCs is driven by sublimation of volatiles as in the case of "classical" bona-fide ecliptic and nearly-isotropic comets (Hsieh et al. 2004; Licandro 2009; Prialnik 2009), is widely accepted.

However, the rapid rotation of $133 \mathrm{P}$ is intriguing and challenging: aside from the mechanism of the solar insolation triggered sublimation, after the discovery of the recurrent activity of the prototype MBC 133P, Hsieh et al. (2004) discussed the possible consequences of its rapid rotation as a possible scenario of the non gas-driven mass loss for this object. According to this scenario if $133 \mathrm{P}$ was an asteroid losing mass from its surface because it rotates on the verge of centripetal instability. The natural consequence of spin-up to instability is a temporary shedding of mass followed by settling into a state just below instability.

The problem with the fast rotation to explain the long-lasting (many months duration) cometary activity is that the material shedding is only an episodic short-term event. However, the fast rotation can influence the gas-driven activity in the interior or at the surface of a small body as it was proposed for some comets and centaurs (Toth \& Lisse 2006). The details of this process are poorly known, although the fast rotation induced shear fracture may open fissures beneath the body surface, which allows more efficient gas and dust outflow from the interior and near-surface regions of the body.

\section{Summary and conclusions}

Our recent analysis of the rotational breakup stability of primitive small bodies of the solar system led to the following conclusions:

(a) We applied more complex models of grain-aggregates to explore the elastomechanical properties of the interior structure of primitive minor bodies of the solar system. We calculated the effective Young's modulus and static tensile strength of grainaggregates and we found a strong decrease of the tensile strength for porosities greater than $\sim 0.7-0.8$. But there are caveats in the understanding the elasto-mechanical properties of primitive small bodies. Recently available models of primitive icy bodies predict both weak (Greenberg et al. 1995) and strong (Sirono \& Greenberg 2000) bulk tensile strengths. Further studies are needed in order to (i) better understand the coagulation and grain growing processes which form primitive small icy bodies and determine their bulk tensile strengths, and (ii) decide whether these bodies are separated according to weaker or stronger bulk tensile strengths.

(b) Using the resulting tensile strengths we constructed the segments of the rotational disintegration classes (allowed, damaged, and forbidden zones) separated by limit curve lines in the effective body radius - rotational period plane.

(c) Most of the comets, centaurs, and small transneptunian objects are stable against the rotational breakup even for the weakest grain-aggregate model (Greenberg et al. 1995). For stronger grain-aggregate (or rocky, stronger icy) models they are surely stable against the rotational disintegration.

(d) There are a few comets, and many centaurs and TNOs in the damaged region, suggesting that comets are fundamentally weaker objects. Some large fast rotators are close to the breakup limit (Davidsson 1999, 2001) unless they have stronger internal strength. 
(e) Main-belt comets are stable - but $133 \mathrm{P} /$ Elst-Pizarro is very close to the breakup limit for the weakest aggregate model but this object is safe for stronger aggregates or rocky materials.

$(f)$ We suggest that Haumea-family could be formed via rotational fission.

\section{Acknowledgements}

I.T. acknowledges the financial support from Konkoly Observatory Budapest, and from the CNRS and CNES during the data analysis and interpretation of the Hubble Space Telescope imaging data at OAMP-LAM, France. C.M.L. acknowledges support from the NASA Deep Impact Extended project for this work.

\section{References}

Belton, M. J. S. 2009, IAU Symposium No. 263 Icy Bodies of the Solar System, Invited.

Biele, J., Ulamec, S., Richter, L., Kührt, E., Knollenberg, Möhlmann, D., \& Rosetta Philae Team 2009, in: Deep Impact as a World Observatory Event: Synergies in Space, Time and Wavelength, ESO Astrophysics Symposia Volume, (Berlin, Heidelberg: Springer), p. 285

Blum, J., Schräpler, R., Davidsson, B. J. R., \& Trigo-Rodriguez, J. M. 2006, ApJ, 652, 1768

Boehnhardt, H. 2004, in: M. C. Festou, H. U. Keller \& H. A. Weaver (eds.), Comets II, Space Science Series, (Tucson: University of Arizona Press), p. 301

Chiang, E. \& Yudin, A. 2009, astro-ph arXiv:0909.2652, 2009 September 14

Davidsson, B. J. R. 1999, Icarus, 142, 525

Davidsson, B. J. R. 2001, Icarus, 149, 375

Dominik, C. \& Tielens, A. G. G. M. 1997, ApJ, 480, 647

Greenberg, J. M., Mizutani, H., \& Yamamoto, T. 1995, A\&SA, 295, L35

Holsapple, K. A. 2007, Icarus, 187, 500

Holsapple, K. A. 2009, Planet. Space Sci., 57, 127

Holsapple, K. A. \& Housen, K. R. 2007, Icarus, 187, 345.

Hsieh, H. H., Jewitt, D., \& Fernández, Y. R. 2004, AJ, 127, 2997

Hsieh, H. H. \& Jewitt, D. 2006, Science, 312, 561

Hsieh, H. H., Jewitt, D., \& Fernández, Y. R. 2009, ApJ, 694, L111

Hsieh, H. H., Jewitt, D., \& Ishiguro, M. 2009, AJ, 137, 157

Jewitt, D. 1992, in: A. Brahic, D.-C. Gerard \& J. Surdej (eds.), Proc. of the 30th Liége International Astrophysical Colloquium, (Liége: University of Liége Press), p. 85

Jewitt, D. 2009, IAU Symposium No. 263: Icy Bodies of the Solar System, Invited.

Jewitt, D. C. \& Meech, K. J. 1988, ApJ, 328, 974

Jewitt, D., Yang, B., \& Haghighipour, N. 2009, AnJ, 137, 4313

Luu, J. X. \& Jewitt, D. C. 1992, AnJ, 104, 2243

Licandro, J. 2009, IAU Symposium No. 263: Icy Bodies of the Solar System, this confence.

Prialnik, D. 2009, in: D. Lazzaro, D. Prialnik, R. Schulz, \& J. A. Fernández (eds.), Icy Bodies of the Solar System, IAU Symposium 263, (Cambridge: Cambridge University Press), this volume.

Richardson, J. E., Melosh, H. J., Lisse, C. M., \& Carcich, B. 2007, Icarus, 190, 357

Richardson, D. C., Schwartz, S. R., Michel, P., \& Walsh, K. J. 2008, BAAS, 40, 498, abstr. 55.02

Richardson, D. C., Michel, P., Walsh, K. J., \& Flynn, K. W. 2009, Planet. Space Sci., 57, 183

Schlichting, H. E. \& Sari, R. 2009, ApJ, 800, 1242

Sirono, S.-I. \& Greenberg, J. M. 2000, Icarus, 145, 230

Toth, I. \& Lisse, C. M. 2006, Icarus, 181, 162

Toth, I., Lamy, P., Weaver, H. A., A'Hearn, M. F., Kaasalainen, M., \& Lowry, S. C. 2006, BAAS, 38, 489, abstr. [06.01]

Toth, I., Lamy, P. L., Weaver, H. A., Noll, K. S., \& Mutchler, M. J. 2008, BAAS, 40, 394, abstr. [05.08] 
Walsh, K. J., Richardson, D. C., \& Michel, P. 2008, BAAS, 40, 498, abstr. 55.03

Weidenschilling, S. J. 2004, in: M. C. Festou, H. U. Keller \& H. A. Weaver (eds.), Comets II, Space Science Series, (Tucson: University of Arizona Press), p. 97

Weissman, P. R., Richardson, D. C., \& Bottke, W. F. 2003, BAAS, 35, 1012, abstr. 47.06

Weissman, P. R., Asphaug, E., \& Lowry, S. C. (2004), in: M. C. Festou, H. U. Keller \& H. A. Weaver (eds.), Comets II, Space Science Series, (Tucson: University of Arizona Press), p. 337

Weissman, P. R. \& Lowry, S. C. 2008, Meteoritics \& Planet. Sci., 43, 1033 